\title{
Kinetic model for anisotropic reactions in amorphous solids
}

Hong, Wei, whong@iastate.edu, lowa State University

\begin{abstract}
When mechanical constraints are present, solid-state reactions often induce deviatoric inelastic strains in addition to volume change. Existing models either attribute such deformation to the plastic flow driven by the stress exceeding nonmeasurable kinetic-dependent yield strength, or need to introduce a deviatoric-stress-dependent chemical potential. By employing the transformation strain to characterize the state of reaction, this letter formulates a kinetic model via averaging the reaction rate at all possible orientations. The model is illustrated through the constrained lithiation-delithiation process of silicon as an example. With just one fitting parameter, the model can quantitatively capture the experimental results. The model only hypothesizes linear kinetics and does not need to introduce kinetic-dependent plasticity or modify basic thermodynamic quantities. This approach can also be applied to other material systems, as well as extended to the nonlinear kinetics of far-from-equilibrium reactions.
\end{abstract}

